\title{
Oral Microbiome: Metagenomics in Oral Health
}

\author{
Ling LU' ${ }^{1}$, Clémentine THABUIS ${ }^{2}$ \\ ${ }^{1}$ Ling LU, Ph.D., R\&D Roquette Management (Shanghai) Co., Ltd., Shanghai, China \\ ${ }^{2}$ Clémentine Thabuis, Ph.D., Nutrition \& Health R\&D, ROQUETTE, Lestrem, France \\ clementine.thabuis@roquette.com
}

\begin{abstract}
The relationship between living organisms in our oral cavity and various diseases, such as dental caries or halitosis, has been well established for many years. In the past, traditional microbiology tools were only possible to deduce the relationships between certain specific bacteria and oral diseases. Today, the evolution of molecular biology, especially of DNA sequencing, and the progress of both bioinformatics and biostatistics allow the detection of uncultivable bacteria that might contribute to the diseases. The interaction among bacterial species can affect biofilm formation and behavior when it is confronted with adverse exogenous factors. Consequently, close investigation of oral microbiota using metagenomics is mandatory to fully understand how to prevent oral diseases related to dysbiosis. Some recent studies have also underlined the putative link between oral and systemic health, emphasizing the importance of understanding oral microbiota and its potential modulations. This review includes (1) the summary of various methodologies for assessing oral microbiota identification, characterization and development, (2) the relationship between oral microbiota and systemic health based on existing animal and human studies, and (3) the possible ways to modulate oral microbiota for positive effects on health.
\end{abstract}

Keywords: metagenomics, oral health, systemic diseases, polyols.

\section{INTRODUCTION}

It is now well known by the general population that our gastrointestinal tract is inhabited, that the human insides are colonized by billions of bacteria, and that we are not alone in our body. People are more and more aware of the presence of various microbiota in or on our body. The most well-known is the gut microbiota, but more recently oral, vaginal and skin microbiota have earned some attention. Some time ago, it was believed that the bacteria were deleterious, that they had to be removed from our lives. Soaps were very strong and bactericidal, toothpastes and mouth rinses aimed to remove $99.9 \%$ of oral bacteria. Nowadays, new care products have been developed to respect human skin, mucosa and their endogenous microflora. Some of them even try to influence these commensal ecosystems in a positive manner. This important change in the general population's way of thinking stems from a better understanding and knowledge of human microbiomes, thanks to high through-put technology such as metagenomics and sequencing. This review will focus on the oral microflora. Firstly, we will describe what metagenomics is in terms of evolution of technology, and then its use in the studies of the influence of living conditions. Secondly, the link between the oral microbiome and systemic health will be discussed. Finally, we will see how the oral microbiota can be modulated by health status or by external factors.

\section{What do We Know about Human Oral Microbiota?}

\section{Metagenomics and Evolution of Technology}

The term microbiome has been defined as the "ecological community of commensal, symbiotic, and pathogenic microorganisms that literally share our body surface and have been all but ignored as determinant of health 
and disease" [17]. The oral microbiome consists of several bacteria in small ecosystems such as the teeth, gums, tongue, lips and cheeks [17]. The human oral microbiome has been widely studied since it can be easily sampled from tooth or saliva [12], contrary to the gut microbiota. Bacterial survival is also better when collected from the oral cavity than from the colon, as living conditions are less harsh in the mouth. Close investigation into this complex biofilm was made possible with the development of molecular biology, and more precisely with the demonstration of high-throughput sequencing technologies [12]. Indeed, traditional bacterial culture studies were not sufficient to gain an accurate view of the biofilm since some species were not able to grow in the set culture conditions. These older technologies were used to isolate approximately 280 bacterial strains from the oral cavity when current molecular techniques can differentiate more than 600 species in the same sample [17]. A global research effort between several international teams led to the compilation of large databases such as The Human Oral Microbiome Database (HOMD) [12], by sharing their results on 16S ribosomal RNA gene sequence analysis. The aim of these large databases is to create a stable taxonomic structure to identify unknown oral taxa according to their 16S rRNA sequence. The larger the database, the more accurate the identification. Other projects aim to define oral microbiota as precisely as possible, such as the NIH Human Microbiome Project [2].

In order to sequence $16 \mathrm{~S}$ rRNA from bacteria, high-throughput sequence analyzers were created in order to obtain faster and less costly sequencings than with the Sanger method. This technology is a combination of molecular material amplification and sequencing. This technology was made possible through improvement in the ability of bioinformatics software to analyze and assign each sequence for a sample. The strengths of these new techniques were compared with those of previous techniques such as microarray [2]. 16S rRNA is found only in prokaryotes and is considered to be the barcode which identifies specific bacteria without the culturable or non-culturable organism problem [2]. Microarray microbe identification is based on the design of specific probes for each bacterium, whereas the metagenomic approach is based on 16S rRNA gene sequence hybridization [2]. Consequently this type of analysis will only find what it is looking for. A study demonstrated that bacterial community profile analyses from $16 \mathrm{~S}$ rRNA pyrosequencing or from microarray assay, produced similar results for the more common taxa at genus level. Nevertheless, pyrosequencing provided for a broader spectrum of taxa identification and greater detection sensitivity for minor species [2]. Firstly, pyrosequencing itself was improved with an increase in the length of rRNA sequences from 100 base pairs (bp) to more than $500 \mathrm{bp}$ for long reads depending on sequencer manufacturer. This increase enabled clearer identification and quantification of common taxa [36] and broader ranging detection of minor taxa [15,60]. With the improvement in sequencing techniques, bioinformatics tools had to be developed in parallel, such as biostatistical analyses. Various bioinformatic techniques were used in order to order and to gather RNA sequences from a biological sample such as OTU (Operational Taxon Unit) [86] or oligotyping [25, 26]. OTU's are poorly-defined clusters of sequences. Resolution is limited. It is better compared to genus, but leaves room for quite a few variations. Oligotypes are based on a mathematical procedure to discriminate between technical noise and biological (true) variation. This method provides for the best possible sequence data resolution [27]. These analyses produced equivalent results for samples that are significantly different, using standard biostatistical tools such as PLS-DA (Projection on Latent Squares - Discriminant Analyses). When there are minor differences between 2 biological samples or if we want to compare 2 time points of a unique ecosystem, biostatics has to be really precise and sensitive. Consequently some specific methods were developed in order to improve the statistical strengths of some analyses such as supervised machine-learning methods [59]. In addition to identification and quantification of taxa in biological samples, $16 \mathrm{~S}$ rRNA sequencing can also be used to identify functional profiles for bacteria communities [45, 56, 67]. Indeed, PICRUSt technology can be used to demonstrate that phylogeny and function are sufficiently correlated to delivery functional prediction for a bacterial community from its 16S rRNA sequence [45]. Today, the resolution and the precision of taxa identification and bacteria quantification are further improved with total DNA sequencing [7]. These broad metagenomics studies also 
improved the functional analyses and prediction of a bacterial community from its DNA sequence [46]. The joint analysis of data sequencing and pictures of biofilm structure will make it possible to understand biofilm community organization and function [54].

\section{Influence of Age and Living Conditions on Oral Microbiome}

These complex and useful tools were at first used to analyze and compare microbial communities in various populations. For instance, very isolated populations were studied in order to understand the development of their salivary microbiota [61]. An African hunter-gatherers tribe shares almost 2 thirds of its oral microorganisms with Western populations. In the same study, Nasidzeet al. also compared populations sharing either geographical localization or way of life. They concluded that similar lifestyles and diet can lead to more similar oral microbiomes than habitat, although larger scale studies would be needed to further investigate these conclusions [61].

The influence of age on microbiota development was also an interesting outcome of metagenomics studies on oral bacteria, as was the influence of early nutrition and parents' oral hygiene [11,14]. A first study demonstrated that the salivary microbiota in children from 3 to 18 years is still developing, and that longitudinal large scale studies would be preferred in order to identify early oral hygiene habits and nutritional behaviors that make for a healthy mouth [14]. The study of early oral microbiota showed that disease risk was not only related to presence of pathogenic bacteria: $10 \%$ of children with dental caries do not exhibit detectable levels of S. mutans for instance [1] and on the contrary, pathogenic bacteria were found in some healthy adults [14]. Delivery mode seems to have an influence as do living conditions (diet, siblings and even presence of pets) [11].

Major lifestyle changes can also significantly affect the oral microbiota. This has been seen among sailors during long sea voyages [99]. Voyages lasting more than 3 months with highly intense work, a different diet and circadian biorhythms in a humid and salty environment, induced a significant decrease in microbial diversity and in microbial metabolism [99]. This study shows that external factors can deeply impact our microbiomes. These results have to be taken into account, together with the concept of resilience. Indeed, it has also been largely demonstrated that if an ecosystem is in equilibrium state, the disturbance has to be highly significant to last a long time; otherwise the microbial system will tend to revert back to its equilibrium state. The influence of antibiotic treatments on oral microbiota has been widely investigated. The effects of various antibiotics on the ecology of both the gut and the oral microbiomes have been studied [98]. The salivary microbiome was found to be significantly more robust, whereas antibiotics negatively affected the fecal microbiome; in particular, health-associated butyrate-producing species became strongly underrepresented; understanding the mechanisms behind the resilience of the oral microbiome toward ecological collapse might have been proven useful in combating microbial dysbiosis elsewhere in the body [98]. Although large scale modifications in the oral microbiome are difficult to observe, there is great inter-individual variation in this ecosystem and even significant intra-day fluctuations, meaning that many precautions have to be taken for observational or interventional clinical trial sampling [73]. Sato et al. concluded that dental plaque sampling is better for preventing intra-day variations and that samples should be collected from the internal face of molars in order to ensure a sufficient quantity of DNA is collected. Nevertheless, due to oral microbiome resilience and inter-individual variations, if a study is investigating slight changes in oral microbiome following a minor disturbance, the time of collection needs to be highly specific, and control groups should be included in the study [73].

The key technical points for investigating oral microbiota development were described in this first section. Monitoring oral microbiota development is not only important in understanding the role of oral microbiota diversity in oral diseases, but it is also strongly linked to the potential influence and relationship that seems to arise between oral microbiota and systemic diseases.

American Research Journal of Dentistry

Page 25 
Oral Microbiome: Metagenomics in Oral Health

\section{Relationship BetWeEn ORAl Microbiota and SyStemic Diseases}

\section{Plausible Mechanisms}

Although the underlying mechanisms between the oral microbiome and systemic diseases have not been fully elucidated, several biologically plausible mechanisms have been put forward [32]. One of the accepted mechanisms is that oral microorganisms evade immune-mediated killing by subverting the host immune response in the oral cavity. They are then disseminated in the systemic circulation through the lesion in the oral cavity, causing bacteraemia and distant site infection [32]. Several oral microorganisms, such as Streptococcus spp., Porphyromonas gingivalis, Fusobacterium nucleatum, Aggregatibacter actinomycetemcomitans, and Prevotella intermedia, have been identified in the blood [55], atheromatous plaque [35], and synovial fluid $[55,83]$, respectively. P. gingivalis, the keystone pathogen in periodontal diseases, is believed to be able to protect itself and other bacteria from immune-mediated killing by subverting the complement function, inactivating antimicrobial peptides, and suppressing microphage endocytosis and nitric oxide-dependent intracellular killing [32]. F. nucleatum, as a potential accessory pathogen, not only invades the host cell itself [33], but also could facilitate the invasion of other pathogens [34]. When these oral bacteria translocate to extra-oral sites, they are likely to stimulate the inflammatory response.

Systemic inflammation plays an important role in the pathogenesis of type 2 diabetes [21], and cardiovascular disease [50]. Triggering of systemic inflammation was put forward as an alternative mechanism for the role of oral bacterial infection in systemic disease development. Chronic exposure to the dysbiotic microbial community leads to a persistently inflammatory environment in the oral cavity. The locally-produced proinflammatory cytokines, such as tumor necrosis factor- $\alpha$ (TNF- $\alpha$ ) and IL-6, are released into the circulation, leading to chronic inflammation, then they stimulate C-reactive protein (CRP) production by liver, further exacerbating systemic inflammation [32]. This hypothesis was supported by the observation of higher serum CRP levels in people with periodontitis compared with healthy controls, and the positive relationship between periodontal pathogens and CRP levels [63].

Another potential mechanism is changes in the composition of the gut microbial community, which has been associated with chronic diseases, such as obesity, metabolic syndrome, type 2 diabetes, and Inflammatory bowel disease (IBD) $[28,72,75,88]$. The Human Microbiome Project (HMP) reported that the oral and gut microbial community could predict each other [18]. Using a high resolution method, Qin et al. compared the gut microbiome in liver cirrhosis and healthy controls [69]. Among enriched species in liver cirrhosis patients, 54\% are species originating from the oral sphere and belonging to the Streptococcus, Fusobacterium, Lactobacillus, Veillonella and Megasphaera groups, suggesting that oral commensal flora invades the gut and becomes overgrown in patients with liver cirrhosis [69]. Animal studies have also shown that oral administration of $P$. gingivalis increases the portion of Bacteroidetes and decreases the portion of Firmicutes in the gut microbiota, and inhibits expression of epithelial tight junction protein in the small intestine [4, 57], resulting in increased gut permeability, which was associated with serum endotoxemia and systemic inflammation. P. gingivalis could only be detected in the jejunum and ileum 1 hour after oral administration, the quantity being decreased at 3 hours [4]. How P. gingivalis leads to changes in the gut microbiota needs to be identified.

\section{Type2 Diabetes}

It has been clearly demonstrated that there is a bi-directional relationship between periodontal disease and type 2 diabetes. On the one hand, the prevalence of periodontal disease is three to four-fold higher in diabetic patients than in healthy controls, which may be due to changes in the microbial community environment and host inflammatory response caused by hyperglycemia [64,65]. On the other hand, baseline periodontal disease was positively associated with a higher risk of incident diabetes [16], and diabetic patients with periodontal disease had worse glycemic control than the diabetic patients without periodontal disease [64].

American Research Journal of Dentistry

Page 26 
A few studies have been conducted to investigate the microbial composition of plaque in type 2 diabetic patients compared with non-diabetic controls, but the results were not consistent $[9,10,23,37]$. Using the checkerboard DNA-DNA hybridization method, Hintaoet al. demonstrated higher Treponemadenticola, Prevotellanigrescens, Streptococcus sanguinis, Streptococcus oralis and Streptococcus intermediuslevels in supragingival plaque samples, and more severe periodontitis, a higher plaque index and a higher prevalence and magnitude of root surface caries in type 2 diabetic patients than non-diabetic controls, however, bacterial distribution in subgingival plaque was not significantly different between the two groups [37]. On the contrary, a higher prevalence of $P$. gingivalis, A. actinomycetemcomitans, and Campylobacter spp. in subgingival plaque samples was observed in Hispanic Americans with type2 diabetes [23]. Similarly, Campus et al. also reported a higher prevalence of P. gingivalis and Tannerella forsythia in type 2 diabetics compared with non-diabetic controls using the polymerase chain reaction (PCR) [9]. With 16s RNA gene sequencing, a study in Brazil reported significant differences in subgingival microbiota when comparing type2 diabetic patients with non-diabetic controls, including higher percentages of TM7, Aggregatibacter, Neisseria, Gemella, Eikenella, Selenomonas, Actinomyces, Capnocytophaga, Fusobacterium, Veillonella and Streptococcus genera [10]. However, some well-established periodontal pathogens, such as T. forsythia and P. gingivalis, were more prevalent in non-diabetic controls than in type 2 diabetic patients [10].

Although type 2 diabetes-related oral microbiota composition is still inconclusive, oral P. gingivalis, $F$. nucleatum and $P$. intermedia treatment-induced periodontitis led to periodontal microbiota dysbiosis, insulin resistance and glucose intolerance, which may be due to impaired adaptive immune response [6]. In addition, lipopolysaccharides from P.gingivaliscould be a major molecular determinant responsible for $P$. gingivalis-aggravated high fat diet-induced insulin resistance [6]. Meanwhile, clinical trials also demonstrated an improvement in glycemic control in type 2 diabetic patients with pre-existing periodontitis following non-surgical periodontal therapy [41, 64]. Combined non-surgical periodontal treatment and antibiotic treatments brought additional benefits [31, 64]. A meta-analysis of clinical trials reported a $0.66 \%$ decrease in HbA1c following non-surgical periodontal therapy alone and a $0.71 \%$ decrease with the addition of antimicrobials in type 2 diabetic patients. However, this was notstatistically significant [41], which may be due to the small sample size.

\section{Atherosclerosis}

Atherosclerosis is a disease in which plaques build up inside the arteries, which leads to the development of cardiovascular diseases, for example myocardial infarction, stroke and coronary heart disease. The hypothesis as to the role of oral microbiota in the development of atherosclerosis is well accepted and supported by the identification of oral pathogens in the atheromatous plaques, including A.actinomycetemcomitans, T. forsythia, P.gingivalis, Non-c serotypes of S.mutans, P. intermedia, and P. nigrescens [29, 35, 58]. In addition, the abundance of Fusobacteriumin the oral cavity was positively associated with levels of cholesterol and LDL-C levels, and Neisseria was negatively associated with HDL-C and ApoAI levels, while Streptococcus was positively associated with HDL-C and ApoAI levels [44].

Among these oral pathogens, P. gingivalis is one of the most prevalent pathogens, and some animal studies have explored the role of $P$. gingivalis in the pathogenic mechanisms of atherosclerotic diseases. Firstly, $P$. gingivalishas to avoid immune-mediated killing. P. gingivaliscan avoid or antagonize TLR4-mediated bacterial activity in macrophages by enzymatically changing the lipid A moiety of its lipopolysaccharides $[32,78]$. This also helps other TLR4-agonistic lipid A-expressing bacteria to avoid TLR4 activation [32, 78]. In the ApoE-/mouse model, oral infection with the inert or antagonistic lipid A-expressing P. gingivalis could lead to vascular inflammation, macrophage infiltration and progression of atherosclerosis [78]. The oral bacteria then spread from the oral mucosa to aortic tissue. Three relocation strategies have been proposed [32]. In addition to a bacteraemic rout, $P$. gingivalis might invade recirculating macrophages and/or dendritic cells and then 
direct these leukocytes to inflammatory aortic tissue, or it might bind to erythrocytes and then release into the circulation [32]. When P. gingivalis arrives in the aortic tissue, it invades the endothelial cells in a FimA fimbriae-dependent manner, where it increases expression of pro-inflammatory molecules [32, 81]. P. gingivalis lipopolysaccharides could stimulate the oxidized LDL-induced macrophage-derived foam cell formation [47, 49]. In addition, the cardiolipin-specific antibody, which could be induced by the epitopes present in P. gingivalis and T.denticola [13], is an autoantibody and cross-reacts with oxLDL [89], suggesting a molecular mimicry strategy is involved in the development of atherosclerosis. Similar molecular mimicry has also recently been reported for A. actinomycetemcomitans [93].

\section{RA}

RA is an autoimmune-mediated chronic inflammatory disease characterized by joint swelling, joint tenderness, destruction of synovial joints and the presence of autoantibodies such as the rheumatoid factor (RF) and anti-citrullinated protein antibody (ACPA), which is recognized as an early serological biomarker of RA, and its level is strongly associated with disease severity $[3,90]$. Although extensive studies have been conducted to explore the relationship between periodontal disease and RA risk since the early $20^{\text {th }}$ century, and a positive relationship has been observed, the underlying mechanisms are still unclear [74]. One hypothesis is that periodontal disease and RA may share some common risk factors, such as smoking, which is an established risk factor for both periodontal disease and RA [74]. Another is from recent research into the oral microbiota, especially the key pathogen in periodontitis, P. gingivalis, which provides further insight into the relationship between periodontal disease and RA. P. gingivalis hasaunique enzyme, Peptidyl Arginine Deiminase (PAD). The arginine gingipains, a proteinase and key virulence factor secreted by $P$. gingivalis, cleave the protein, such as bacterial protein and human fibrinogen and $\alpha$-enolase, resulting in a peptide with carboxy-terminal arginine, which is then citrullinated by P. gingivalis-derived PAD, and subsequently generatesneoepitopes [95]. This potential mechanism could trigger autoantibody production (i.e. ACPAs), which could form immune complexes with the citrullinated host protein and exacerbate inflammation in RA [32]. Infection of mice with wild type $P$. gingivalis significantly increased levels of autoantibodies to collagen type II and citrullinated epitopes, and this effect depended on the expression of $P$. gingivalis-derived PAD [53].

In human studies, the DNA of periodontal bacteria, P. intermedia, P. gingivalis, F. nucleatum and $S$. proteamaculans, was detected in synovial fluid of patients with RA and periodontal disease [55, 83]. In addition, Anaeroglobusgerminatus, Prevotella, Leptrotrichiaand Tannerella species have been associated with either RA-related autoantibodies or early-onset RA [74]. Due to the limited sample size of previous studies, these findings need to be replicated in larger population studies, and the hypothesis that there is synergistic interaction among these bacterial species requires further exploration.

\section{IBD}

IBD groups a number of conditions leading to chronic inflammation in the gastrointestinal (GI) tract, mainly ulcerative colitis (UC) and Crohn's disease (CD). Extensive evidence suggests that dysbiotic gut microbiota is involved in the pathogenesis of IBD [28, 72]. As mentioned above, oral microbiota is believed to be able to change the composition of the gut microbiota. Therefore, it is not surprising to observe an association between the oral microbiota and IBD.

The specific oral Campylobacter concisus strain, enteric invasive C. concisus (EICC) strain, was detected in 50\% IBD patients, but not in control patients, although this difference was not significant [40]. Lesser microbial diversity in tongue swab samples was observed in the CD patients, but not in UC patients. This was mainly attributed to the loss of Fusobacteria and Firmicutes, and to an increase in Spirochaetes, Synergistetes and Bacteroidetes [19]. Similarly, an increase in Bacteroidetes and decrease in Proteobacteria in the saliva samples of IBD patients was also reported in a recent study, and the results showed that Streptococcus, Prevotella, Neisseria, Haemophilus, 
Veillonella, and Gemella contributed to the dysbiosis of salivary microbiota in IBD patients [71]. There are only few animal studies exploring the underlying mechanism. In a mouse model of dextran sodium sulfate-induced colitis, intravenous administration of the highly-virulent S.mutans strain isolated from UC patients, but not the standard S. mutans strain,was seen to aggravate colitis [43]. In keeping with this finding, S. mutans could also colonize in the hepatocytes of liversin the colitis mice, and increased expression of interferon-Y in the liver was observed [74].

The relationship between oral microbiota and systemic health has been illustrated through various examples. An attempt will be made to understand how our oral microbiota could be influenced in the next part of this review.

\section{How to Modulate Our Oral Microbiome?}

\section{Disease-Mediated Modulation}

As previously described, the oral bacteria ecosystem is extremely strong and difficult to modulate whether positively or negatively. Chronic diseases are nevertheless able to do this. If we focus on the oral domain, it has been demonstrated that even a few volunteers were sufficient to discriminate Chinese adults with or without gingivitis [39]. A number of organisms were strongly associated with the gingivitis phenotype such as Leptotrichia and Selomonas, which could constitute good biomarkers for onset of gingivitis [39]. It is known that the bacterial biofilm is able to recover rapidly after professional prophylaxis [82], the order of bacteria succession regrowth being of importance for the "health status" of the future microbiome. The prevalence of species strongly correlated with periodontal pathogenesis was decreased during early subgingival recolonization. Indeed, recolonization order was the same in periodontal health and disease, suggesting that many factors have to be taken into account in order to orient the redeveloping biofilm towards "healthy" or "sick" status. Consequently, it seems possible to promote beneficial microbiome regrowth by clearly understanding the mechanisms that control the succession of bacterial recolonization [82]. Larger studies were performed to characterize the microbiota linked to periodontitis. The analyses in various countries identifiedthe same bacterial strains. Prevotella, Porphyromonas, and Treponema were mainly linked to this infectious state [87]. The technological improvements in pyrosequencing and the increasing power of biostatics made it possible to develop functional analyses for the bacteria identified. Further analysis of these bacteria linked to chronic periodontitis was useful in shedding light on functional genes and metabolic pathways that are particularly activated during acute disease phases [94]. These particular pathways were involved in bacterial chemotaxis, flagellar assembly and toxin biosynthesis.

One of the most frequently studied oral diseases remains dental caries. Conventional techniques were useful to identify and characterize some bacteria particularly linked to tooth decay such as S. mutans and Lactobacillus spp. [1]. Pyrosequencing enabled the identification and quantification of more strains present in healthy volunteers or in volunteers with active caries. Four different phyla were especially linked to dental caries such as Bacteroidetes, Firmicutes, Fusobacteria and Proteobacteria [97]. However, the non-systematic pathogenic nature of $S$. mutanswas also revealed [14]. Disease is not linked to ONE bacterial presence but to multifactorial causes [92]. Consequently the microbial interaction between bacteria, and their way of interacting with external factors is mandatory to understand why they become pathogenic or why they remain harmless.

A harmless but socially deleterious disease has also been widely studied in order to be countered; indeed halitosis can be a genuinely embarrassing and chronic disease. Halitosis was linked to the presence of specific bacteria in saliva in Chinese children [70]. 16S rRNA gene pyrosequencing and metagenomic sequencing were used to examine oral microbial composition and its functional variations in children with halitosis. The tongue coating of subjects with halitosis was morebacteria-rich than that of healthy subjects. The relative abundance and prevalence of Leptotrichiawadei and Peptostreptococcus stomatis were higher in tongue coating samples 
from children with halitosis as was Prevotellashahii in their saliva. It seems that tongue and saliva communities did not follow the same development in spite of their geographical proximity. Moreover, functional interpretation of $16 \mathrm{~S}$ sequencing places the emphasis on the correlation between the presence of genes involved in polyketide metabolism and in hydrogen sulfide-related metabolic pathways with halitosis, suggesting that there was higher microbial production and less usage of $\mathrm{H}_{2} \mathrm{~S}$ in subjects with halitosis [70].

\section{Modulation Induced by Exogenous Factors}

If we consider the various external factors likely to have an effect on dental health, we have to consider cigarette smoking. It is already known that nicotine may have a strong impact on the growth of bacteria involved in tooth decay [38, 48]. In addition, an observational study looked at the oral microbiota of smokers among American adults [96]. Overall oral microbiome composition differed between former smokers and current non-smokers based on the relative abundance of some taxa and genera (Proteobacteria, Capnocytophaga, Peptostreptococcus and Leptotrichia were depleted, while Atopobium and Streptococcus were enriched during smoking) [96]. Functional analysis of the sequencing showed that bacterial genera depletion by smoking was related to carbohydrate and energy metabolism, and to xenobiotic metabolism [96].

We have demonstrated, using several examples, that oral microbiota could be negatively modulated by exogenous and endogenous factors. The challenge to be faced now is to positively modulate this microbiome in order to keep our mouth and our whole body in general healthy condition using nutritional vectors.

The effects of nutritional supplementation on oral microbiota have been widely studied, but mainly using traditional microbiology techniques. Many plant extracts were tested on bacterial strains associated with dental caries, mainly S. mutans. Where effects were observed, these extracts were considered to be bacteriostatic and anti-cariogenic. Various administration methods such as mouth rinses, candies and chewing-gums were used in interventional clinical trials or simple extracts in in-vitro studies $[8,66,68]$.

Some preliminary studies were conducted in order to influence mouth microbiota using bacteria known to be probiotic. Indeed, dental diseases can be considered as microbial imbalances resulting from a shift from healthy oral microbiota to dysbiosis, and a shift towards communities which are dominated by acidogenic and acid-tolerant gram-positive bacteria in the case of tooth decay [5]. It has been believed that probiotic bacteria such as Lactobacillus or Bifidobacterium could be effective in restoring balance to the oral microbiota, thus preventing dental caries or inflammatory oral diseases such as gingivitis or periodontitis. The putative beneficial effects of probiotics on bad breath have also been evaluated, but further evidence is needed to fully explore the potential of probiotics for preventing bad breath [5]. Supplementation using L. reuteri was administered to healthy subjects for 12 weeks [77]. It was demonstrated that the supplementation was useful in implementing this probiotic in the saliva microbiota during the intervention, however L. reuteri was washed-out at the end of the study. Consequently, modulation of oral microbiota using probiotics was only temporary and did not last, showing once again the resilience of healthy oral microbiota [77]. Another attempt was made in a longitudinal analysis of oral and more particularly salivary microbiome, where volunteers received supplementation in the form ofa commercial probiotic product containing milk fermented with S. thermophilus, L. bulgaricus and L. paracasei [15]. A significant change in microbial community was observed mainly on Streptococcus and Actinomyces genera. The presence of the Lactobacillus strain administered was not detected, whichsupports the hypothesis that colonization is not a requisite for a modulation of the microbiota. A microbial ecosystem can be influenced without intrusion [15]. Nevertheless, this supplementation was acute and the long lasting effect on microbiota was not investigated. Consequently, we may doubt that this modulation is persistent. From these studies on probiotic supplementation, we learned that salivary microbiome modulations are not easy to observe and to characterize. Protocols need to be highly specific, as do sampling and analyses. It should also be noted that dental plaque microbiota is more sensitive to shifts than the salivary ecosystem.

American Research Journal of Dentistry

Page 30 
Interactions between food products and microbiota have been widely studied, with a special focus on the gut microbiome and fiber interaction [76]. Concerning the oral flora, a close look was taken on the health effects of sugar-free confectionary components such as polyols. Indeed, sugar alcohols are known to have beneficial effects on oral health in that they are poorly or not metabolized by oral bacteria and that they enhance saliva [51,52, 84, 85, 91]. They showed positive effects on remineralization [20,22] and gingivitis [42]. Their inhibitory effects on oral bacteria were also tested in-vivo and in-vitro [24, 30, 80]. Xylitol is the most frequently studied polyol as much in-vivo as in-vitro, using various vectors and doses [62]. There are fewer publications on overall modulation of oral microbiotaby food products than on the gut microbiota. The scientific community has focused on the impact of diseases or of drugs on this specific ecosystem but not of food components. The only preliminary study for the time being was conducted by Soderling et al. [79] and did not conclude on any effect from xylitol gum supplementation on salivary microbiota in children. Nevertheless, the choice was made to study the salivary microbiome, whereas it is now known that microflora modulations are more difficult to observe in saliva during interventional studies than in dental plaque. For short-term exposure, only 5 weeks' consumption, the most sensitive ecosystem should have been chosen. Moreover, the control group included children chewing sorbitol gum which could also impact oral microbiota, as it is the same kind of molecule as xylitol. Consequently, a more inert placebo could have made it possible to see some changes in the microbiota, even for 5 weeks' exposure. Compared with other clinical interventions on gums, $6 \mathrm{~g}$ of xylitol per day is a commonly used dosage that has already demonstrated significant effects, and compared with other metagenomics study in dental health, the statistical power should have been sufficient, $n=35$ to 38 per group, for observing biologically-relevant modulation in the oral microbiota.

To study the impact of food products on the oral microbiome, as we expect slight modulations due to the resilience of this ecosystem, it would be preferable to work on large standardized populations with an accurate placebo control, modern high-throughput technology, and strong biostatistics tools with a specific focus on dental plaque microbiota. The sampling procedure and timing also need to be really appropriated.

\section{CONCLUSION}

It has been shown that investigating changes in the oral microbiota can actually be very difficult due to interindividual and even intraday variations. Consequently, clinical methodologies have to be firmly established and the latesthigh-throughput metagenomics, bioinformatics and statistics toolsshould be used. There is growing evidence to suggest a relationship between the oral microbiota especially periodontal disease bacteria, and systemic diseases. However, the sample size of most of these studies was too small to be able to come to a conclusion. More large longitudinal studies and clinical trials are needed. In addition, whether the dysbiosis of oral microbiota is a cause or a consequence of systemic diseases needs to be clarified. To explore etiopathogenesis, multiple "omics" methods, like metagenomics, metatranscriptomics, and metaproteomics, could be applied. If the causal relationship is confirmed, correcting the dysbiotic oral microbiota will not only be a means of preventing and controlling oral diseases, but will also provide additional benefitsin the treatment of systemic diseases.

\section{Acknowledgements}

The authors thank Caroline PERREAU for formatting the manuscript. They also thank Coralie DESMIDT for language editing.

\section{REFERENCE}

1. Aas JA, Griffen AL, Dardis SR, Lee AM, Olsen I, Dewhirst FE, Leys EJ, Paster BJ. Bacteria of dental caries in primary and permanent teeth in children and young adults. J.Clin.Microbiol. 2008; 46:1407-1417

2. Ahn J, Yang L, Paster BJ, Ganly I, Morris L, Pei Z, Hayes RB. Oral microbiome profiles: 16S rRNA pyrosequencing and microarray assay comparison. PLoS.One. 2011; 6:e22788

American Research Journal of Dentistry

Page 31 
3. Aletaha D, Neogi T, Silman AJ, Funovits J, Felson DT, Bingham CO, III, Birnbaum NS, Burmester GR, Bykerk VP, Cohen MD, Combe B, Costenbader KH, Dougados M, Emery P, Ferraccioli G, Hazes JM, Hobbs K, Huizinga TW, Kavanaugh A, Kay J, Kvien TK, Laing T, Mease P, Menard HA, Moreland LW, Naden RL, Pincus T, Smolen JS, Stanislawska-Biernat E, Symmons D, Tak PP, Upchurch KS, Vencovsky J, Wolfe F, Hawker G. 2010 Rheumatoid arthritis classification criteria: an American College of Rheumatology/European League Against Rheumatism collaborative initiative. Arthritis Rheum. 2010; 62:2569-2581

4. Arimatsu K, Yamada H, Miyazawa H, Minagawa T, Nakajima M, Ryder MI, Gotoh K, Motooka D, Nakamura S, Iida T, Yamazaki K. Oral pathobiont induces systemic inflammation and metabolic changes associated with alteration of gut microbiota. Sci.Rep. 2014; 4:4828

5. Bizzini B, Pizzo G, Scapagnini G, Nuzzo D, Vasto S. Probiotics and oral health. Curr.Pharm.Des 2012; 18:55225531

6. Blasco-Baque V, Garidou L, Pomie C, Escoula Q, Loubieres P, Le Gall-David S, Lemaitre M, Nicolas S, Klopp P, Waget A, Azalbert V, Colom A, Bonnaure-Mallet M, Kemoun P, Serino M, Burcelin R. Periodontitis induced by Porphyromonas gingivalis drives periodontal microbiota dysbiosis and insulin resistance via an impaired adaptive immune response. Gut 2017; 66:872-885

7. Blottiere HM, de Vos WM, Ehrlich SD, Dore J. Human intestinal metagenomics: state of the art and future. Curr.Opin.Microbiol. 2013; 16:232-239

8. Campus G, Cagetti MG, Cocco F, Sale S, Sacco G, Strohmenger L, Lingstrom P. Effect of a sugar-free chewing gum containing magnolia bark extract on different variables related to caries and gingivitis: a randomized controlled intervention trial. Caries Res. 2011; 45:393-399

9. Campus G, Salem A, Uzzau S, Baldoni E, Tonolo G. Diabetes and periodontal disease: a case-control study. J.Periodontol. 2005; 76:418-425

10. Casarin RC, Barbagallo A, Meulman T, Santos VR, Sallum EA, Nociti FH, Duarte PM, Casati MZ, Goncalves RB. Subgingival biodiversity in subjects with uncontrolled type-2 diabetes and chronic periodontitis. J.Periodontal Res. 2013; 48:30-36

11. Cephas KD, Kim J, Mathai RA, Barry KA, Dowd SE, Meline BS, Swanson KS. Comparative analysis of salivary bacterial microbiome diversity in edentulous infants and their mothers or primary care givers using pyrosequencing. PLoS.One. 2011; 6:e23503

12. Chen T, Yu WH, Izard J, Baranova OV, Lakshmanan A, Dewhirst FE. The Human Oral Microbiome Database: a web accessible resource for investigating oral microbe taxonomic and genomic information. Database. (Oxford) 2010; 2010:baq013

13. Chen YW, Nagasawa T, Wara-aswapati N, Ushida Y, Wang D, Takeuchi Y, Kobayashi H, Umeda M, Inoue Y, Iwai T, Ishikawa I, Izumi Y. Association between periodontitis and anti-cardiolipin antibodies in Buerger disease. J.Clin.Periodontol. 2009; 36:830-835

14. Crielaard W, Zaura E, Schuller AA, Huse SM, Montijn RC, Keijser BJ. Exploring the oral microbiota of children at various developmental stages of their dentition in the relation to their oral health. BMC.Med.Genomics $2011 ; 4: 22$

15. Dassi E, Ballarini A, Covello G, Quattrone A, Jousson O, De S, V, Bertorelli R, Denti MA, Segata N. Enhanced microbial diversity in the saliva microbiome induced by short-term probiotic intake revealed by $16 \mathrm{~S}$ rRNA sequencing on the IonTorrent PGM platform. J.Biotechnol. 2014; 
16. Demmer RT, Jacobs DR, Jr., Desvarieux M. Periodontal disease and incident type 2 diabetes: results from the First National Health and Nutrition Examination Survey and its epidemiologic follow-up study. Diabetes Care 2008; 31:1373-1379

17. Dewhirst FE, Chen T, Izard J, Paster BJ, Tanner AC, Yu WH, Lakshmanan A, Wade WG. The human oral microbiome. J.Bacteriol. 2010; 192:5002-5017

18. Ding T, Schloss PD. Dynamics and associations of microbial community types across the human body. Nature 2014; 509:357-360

19. Docktor MJ, Paster BJ, Abramowicz S, Ingram J, Wang YE, Correll M, Jiang H, Cotton SL, Kokaras AS, Bousvaros A. Alterations in diversity of the oral microbiome in pediatric inflammatory bowel disease. Inflamm.Bowel. Dis. 2012; 18:935-942

20. Dodds MW, Chidichimo D, Haas MS. Delivery of active agents from chewing gum for improved remineralization. Adv.Dent.Res. 2012; 24:58-62

21. Donath MY. Targeting inflammation in the treatment of type 2 diabetes: time to start. Nat.Rev.Drug Discov. 2014; 13:465-476

22. Dong Y, Yin W, Hu D, Zhang X, Xu L, Dodds WJ, Tian M. Remineralization of early caries by chewing sugarfree gum: a clinical study using quantitative light-induced fluorescence. Am.J.Dent. 2014; 27:291-295

23. Ebersole JL, Holt SC, Hansard R, Novak MJ. Microbiologic and immunologic characteristics of periodontal disease in Hispanic americans with type 2 diabetes. J.Periodontol. 2008; 79:637-646

24. Edwardsson S, Birkhed D, Mejare B. Acid production from Lycasin, maltitol, sorbitol and xylitol by oral streptococci and lactobacilli. Acta Odontol.Scand. 1977; 35:257-263

25. Eren AM, Borisy GG, Huse SM, Mark Welch JL. Oligotyping analysis of the human oral microbiome. Proc. Natl. Acad.Sci.U.S.A 2014; 111:E2875-E2884

26. Eren AM, Maignien L, Sul WJ, Murphy LG, Grim SL, Morrison HG, Sogin ML. Oligotyping: Differentiating between closely related microbial taxa using 16S rRNA gene data. Methods Ecol.Evol. 2013; 4

27. Eren AM, Morrison HG, Lescault PJ, Reveillaud J, Vineis JH, Sogin ML. Minimum entropy decomposition: unsupervised oligotyping for sensitive partitioning of high-throughput marker gene sequences. ISME.J. 2015; 9:968-979

28. Frank DN, St Amand AL, Feldman RA, Boedeker EC, Harpaz N, Pace NR. Molecular-phylogenetic characterization of microbial community imbalances in human inflammatory bowel diseases. Proc.Natl. Acad.Sci.U.S.A 2007; 104:13780-13785

29. Gaetti-Jardim E Jr, Marcelino SL, Feitosa AC, Romito GA, Avila-Campos MJ. Quantitative detection of periodontopathic bacteria in atherosclerotic plaques from coronary arteries. J.Med.Microbiol. 2009; 58:1568-1575

30. Giertsen E, Arthur RA, Guggenheim B. Effects of xylitol on survival of mutans streptococci in mixed-sixspecies in vitro biofilms modelling supragingival plaque. Caries Res. 2011; 45:31-39

31. Grossi SG, Skrepcinski FB, DeCaro T, Robertson DC, Ho AW, Dunford RG, Genco RJ. Treatment of periodontal disease in diabetics reduces glycated hemoglobin. J.Periodontol. 1997; 68:713-719

32. Hajishengallis G. Periodontitis: from microbial immune subversion to systemic inflammation. Nat.Rev. Immunol. 2015; 15:30-44 
33. Han YW, Shi W, Huang GT, Kinder HS, Park NH, Kuramitsu H, Genco RJ. Interactions between periodontal bacteria and human oral epithelial cells: Fusobacterium nucleatum adheres to and invades epithelial cells. Infect.Immun. 2000; 68:3140-3146

34. Han YW, Wang X. Mobile microbiome: oral bacteria in extra-oral infections and inflammation. J.Dent.Res. 2013; 92:485-491

35. Haraszthy VI, Zambon JJ, Trevisan M, Zeid M, Genco RJ. Identification of periodontal pathogens in atheromatous plaques. J.Periodontol. 2000; 71:1554-1560

36. Hasan NA, Young BA, Minard-Smith AT, Saeed K, Li H, Heizer EM, McMillan NJ, Isom R, Abdullah AS, Bornman DM, Faith SA, Choi SY, Dickens ML, Cebula TA, Colwell RR. Microbial community profiling of human saliva using shotgun metagenomic sequencing. PLoS.One. 2014; 9:e97699

37. Hintao J, Teanpaisan R, Chongsuvivatwong V, Ratarasan C, Dahlen G. The microbiological profiles of saliva, supragingival and subgingival plaque and dental caries in adults with and without type 2 diabetes mellitus. Oral Microbiol.Immunol. 2007; 22:175-181

38. Huang R, Li M, Gregory RL. Effect of nicotine on growth and metabolism of Streptococcus mutans. Eur.J.Oral Sci. 2012; 120:319-325

39. Huang S, Yang F, Zeng X, Chen J, Li R, Wen T, Li C, Wei W, Liu J, Chen L, Davis C, Xu J. Preliminary characterization of the oral microbiota of Chinese adults with and without gingivitis. BMC.Oral Health 2011; 11:33

40. Ismail Y, Mahendran V, Octavia S, Day AS, Riordan SM, Grimm MC, Lan R, Lemberg D, Tran TA, Zhang L. Investigation of the enteric pathogenic potential of oral Campylobacter concisus strains isolated from patients with inflammatory bowel disease. PLoS.One. 2012; 7:e38217

41. Janket SJ, Wightman A, Baird AE, Van Dyke TE, Jones JA. Does periodontal treatment improve glycemic control in diabetic patients? A meta-analysis of intervention studies. J.Dent.Res. 2005; 84:1154-1159

42. Keukenmeester R, Slot D, Putt M, Van der WG. The effect of sugar-free chewing gum on plaque and clinical parameters of gingival inflammation: a systematic review. Int.J.Dent.Hyg. 2012;

43. Kojima A, Nakano K, Wada K, Takahashi H, Katayama K, Yoneda M, Higurashi T, Nomura R, Hokamura K, Muranaka Y, Matsuhashi N, Umemura K, Kamisaki Y, Nakajima A, Ooshima T. Infection of specific strains of Streptococcus mutans, oral bacteria, confers a risk of ulcerative colitis. Sci.Rep. 2012; 2:332

44. Koren O, Spor A, Felin J, Fak F, Stombaugh J, Tremaroli V, Behre CJ, Knight R, Fagerberg B, Ley RE, Backhed F. Human oral, gut, and plaque microbiota in patients with atherosclerosis. Proc.Natl.Acad.Sci.U.S.A 2011; 108 Suppl 1:4592-4598

45. Langille MG, Zaneveld J, Caporaso JG, McDonald D, Knights D, Reyes JA, Clemente JC, Burkepile DE, Vega Thurber RL, Knight R, Beiko RG, Huttenhower C. Predictive functional profiling of microbial communities using 16S rRNA marker gene sequences. Nat.Biotechnol. 2013; 31:814-821

46. Larraufie P, de WT, Potocki-Veronese G, Blottiere HM, Dore J. Functional metagenomics to decipher foodmicrobe-host crosstalk. Proc.Nutr.Soc. 2015; 74:1-4

47. Lei L, Li H, Yan F, Li Y, Xiao Y. Porphyromonas gingivalis lipopolysaccharide alters atherosclerotic-related gene expression in oxidized low-density-lipoprotein-induced macrophages and foam cells. J.Periodontal Res. 2011; 46:427-437

48. Li MY, Huang RJ, Zhou XD, Gregory RL. Role of sortase in Streptococcus mutans under the effect of nicotine. Int.J.Oral Sci. 2013; 5:206-211 
Oral Microbiome: Metagenomics in Oral Health

49. Liang DY, Liu F, Chen JX, He XL, Zhou YL, Ge BX, Luo LJ. Porphyromonas gingivalis infected macrophages upregulate CD36 expression via ERK/NF-kappaB pathway. Cell Signal. 2016; 28:1292-1303

50. Libby P. Inflammation and cardiovascular disease mechanisms. Am.J.Clin.Nutr. 2006; 83:456S-460S

51. Makinen KK. Sugar alcohols, caries incidence, and remineralization of caries lesions: a literature review. Int.J.Dent. 2010; 2010:981072

52. Makinen KK, Soderling E, Isokangas P, Tenovuo J, Tiekso J. Oral biochemical status and depression of Streptococcus mutans in children during 24- to 36-month use of xylitol chewing gum. Caries Res. 1989; 23:261-267

53. Maresz KJ, Hellvard A, Sroka A, Adamowicz K, Bielecka E, Koziel J, Gawron K, Mizgalska D, Marcinska KA, Benedyk M, Pyrc K, Quirke AM, Jonsson R, Alzabin S, Venables PJ, Nguyen KA, Mydel P, Potempa J. Porphyromonas gingivalis facilitates the development and progression of destructive arthritis through its unique bacterial peptidylarginine deiminase (PAD). PLoS.Pathog. 2013; 9:e1003627

54. Mark Welch JL, Rossetti BJ, Rieken CW, Dewhirst FE, Borisy GG. Biogeography of a human oral microbiome at the micron scale. Proc.Natl.Acad.Sci.U.S.A 2016; 113:E791-E800

55. Martinez-Martinez RE, Abud-Mendoza C, Patino-Marin N, Rizo-Rodriguez JC, Little JW, Loyola-Rodriguez JP. Detection of periodontal bacterial DNA in serum and synovial fluid in refractory rheumatoid arthritis patients. J.Clin.Periodontol. 2009; 36:1004-1010

56. McLean JS. Advancements toward a systems level understanding of the human oral microbiome. Front Cell Infect.Microbiol. 2014; 4:98

57. Nakajima M, Arimatsu K, Kato T, Matsuda Y, Minagawa T, Takahashi N, Ohno H, Yamazaki K. Oral Administration of P. gingivalis Induces Dysbiosis of Gut Microbiota and Impaired Barrier Function Leading to Dissemination of Enterobacteria to the Liver. PLoS.One. 2015; 10:e0134234

58. Nakano K, Nemoto H, Nomura R, Homma H, Yoshioka H, Shudo Y, Hata H, Toda K, Taniguchi K, Amano A, Ooshima T. Serotype distribution of Streptococcus mutans a pathogen of dental caries in cardiovascular specimens from Japanese patients. J.Med.Microbiol. 2007; 56:551-556

59. Nakano Y, Takeshita T, Kamio N, Shiota S, Shibata Y, Suzuki N, Yoneda M, Hirofuji T, Yamashita Y. Supervised machine learning-based classification of oral malodor based on the microbiota in saliva samples. Artif. Intell.Med. 2014; 60:97-101

60. Nasidze I, Li J, Quinque D, Tang K, Stoneking M. Global diversity in the human salivary microbiome. Genome Res. 2009; 19:636-643

61. Nasidze I, Li J, Schroeder R, Creasey JL, Li M, Stoneking M. High diversity of the saliva microbiome in Batwa Pygmies. PLoS.One. 2011; 6:e23352

62. Nayak PA, Nayak UA, Khandelwal V. The effect of xylitol on dental caries and oral flora. Clin.Cosmet.Investig. Dent. 2014; 6:89-94

63. Noack B, Genco RJ, Trevisan M, Grossi S, Zambon JJ, De NE. Periodontal infections contribute to elevated systemic C-reactive protein level. J.Periodontol. 2001; 72:1221-1227

64. Ohlrich EJ, Cullinan MP, Leichter JW. Diabetes, periodontitis, and the subgingival microbiota. J.Oral Microbiol. 2010; 2

65. Ojima M, Takeda M, Yoshioka H, Nomura M, Tanaka N, Kato T, Shizukuishi S, Amano A. Relationship of periodontal bacterium genotypic variations with periodontitis in type 2 diabetic patients. Diabetes Care 2005; 28:433-434

American Research Journal of Dentistry

Page 35 
66. Ooshima T, Osaka Y, Sasaki H, Osawa K, Yasuda H, Matsumura M, Sobue S, Matsumoto M. Caries inhibitory activity of cacao bean husk extract in in-vitro and animal experiments. Arch.Oral Biol. 2000; 45:639-645

67. Parks DH, Tyson GW, Hugenholtz P, Beiko RG. STAMP: statistical analysis of taxonomic and functional profiles. Bioinformatics. 2014; 30:3123-3124

68. Percival RS, Devine DA, Duggal MS, Chartron S, Marsh PD. The effect of cocoa polyphenols on the growth, metabolism, and biofilm formation by Streptococcus mutans and Streptococcus sanguinis. Eur.J.Oral Sci. 2006; 114:343-348

69. Qin N, Yang F, Li A, Prifti E, Chen Y, Shao L, Guo J, Le CE, Yao J, Wu L, Zhou J, Ni S, Liu L, Pons N, Batto JM, Kennedy SP, Leonard P, Yuan C, Ding W, Chen Y, Hu X, Zheng B, Qian G, Xu W, Ehrlich SD, Zheng S, Li L. Alterations of the human gut microbiome in liver cirrhosis. Nature 2014; 513:59-64

70. Ren W, Xun Z, Wang Z, Zhang Q, Liu X, Zheng H, Zhang Q, Zhang Y, Zhang L, Wu C, Zheng S, Qin N, Ehrlich SD, Li Y, He X, Xu T, Chen T, Chen F. Tongue Coating and the Salivary Microbial Communities Vary in Children with Halitosis. Sci.Rep. 2016; 6:24481

71. Said HS, Suda W, Nakagome S, Chinen H, Oshima K, Kim S, Kimura R, Iraha A, Ishida H, Fujita J, Mano S, Morita H, Dohi T, Oota H, Hattori M. Dysbiosis of salivary microbiota in inflammatory bowel disease and its association with oral immunological biomarkers. DNA Res. 2014; 21:15-25

72. Sartor RB. Microbial influences in inflammatory bowel diseases. Gastroenterology 2008; 134:577-594

73. Sato Y, Yamagishi J, Yamashita R, Shinozaki N, Ye B, Yamada T, Yamamoto M, Nagasaki M, Tsuboi A. InterIndividual Differences in the Oral Bacteriome Are Greater than Intra-Day Fluctuations in Individuals. PLoS. One. 2015; 10:e0131607

74. Scher JU, Bretz WA, Abramson SB. Periodontal disease and subgingival microbiota as contributors for rheumatoid arthritis pathogenesis: modifiable risk factors? Curr.Opin.Rheumatol. 2014; 26:424-429

75. Sekirov I, Russell SL, Antunes LC, Finlay BB. Gut microbiota in health and disease. Physiol Rev. 2010; 90:859904

76. Simpson HL, Campbell BJ. Review article: dietary fibre-microbiota interactions. Aliment.Pharmacol.Ther. 2015; 42:158-179

77. Sinkiewicz G, Cronholm S, Ljunggren L, Dahlen G, Bratthall G. Influence of dietary supplementation with Lactobacillus reuteri on the oral flora of healthy subjects. Swed.Dent.J. 2010; 34:197-206

78. Slocum C, Coats SR, Hua N, Kramer C, Papadopoulos G, Weinberg EO, Gudino CV, Hamilton JA, Darveau RP, Genco CA. Distinct lipid a moieties contribute to pathogen-induced site-specific vascular inflammation. PLoS.Pathog. 2014; 10:e1004215

79. Soderling E, ElSalhy M, Honkala E, Fontana M, Flannagan S, Eckert G, Kokaras A, Paster B, Tolvanen M, Honkala S. Effects of short-term xylitol gum chewing on the oral microbiome. Clin.Oral Investig. 2015; 19:237-244

80. Soderling E, Makinen KK, Chen CY, Pape HR, Jr., Loesche W, Makinen PL. Effect of sorbitol, xylitol, and xylitol/ sorbitol chewing gums on dental plaque. Caries Res. 1989; 23:378-384

81. Takahashi N, Nyvad B. Caries Ecology Revisited: Microbial Dynamics and the Caries Process. Caries Res 2008; 42:409-418 
Oral Microbiome: Metagenomics in Oral Health

82. Teles FR, Teles RP, Uzel NG, Song XQ, Torresyap G, Socransky SS, Haffajee AD. Early microbial succession in redeveloping dental biofilms in periodontal health and disease. J.Periodontal Res. 2012; 47:95-104

83. Temoin S, Chakaki A, Askari A, El-Halaby A, Fitzgerald S, Marcus RE, Han YW, Bissada NF. Identification of oral bacterial DNA in synovial fluid of patients with arthritis with native and failed prosthetic joints. J.Clin. Rheumatol. 2012; 18:117-121

84. Thabuis C, Cheng CY, Wang X, Pochat M, Han A, Miller L, Wils D, Guerin-Deremaux L. Maltitol and Xylitol Sweetened Chewing-Gums Could Modulate Salivary Parameters Involved in Dental Caries Prevention. JBR Journal of Interdisciplinary Medicine and Dental Science 2016; 4:doi:10.4172/2376-032X.1000191

85. Thabuis C, Rodriguez B, Gala T, Salvi A, Parashuraman M, Wils D, Guerin-Deremaux L. Evaluation of glycemic and insulinemic responses of maltitol in Indian healthy volunteers. Int J Diabetes Dev Ctries 2015;

86. Tikhonov M, Leach RW, Wingreen NS. Interpreting 16S metagenomic data without clustering to achieve sub-OTU resolution. ISME.J. 2015; 9:68-80

87. Tsai CY, Tang CY, Tan TS, Chen KH, Liao KH, Liou ML. Subgingival microbiota in individuals with severe chronic periodontitis. J.Microbiol.Immunol.Infect. 2016;

88. Ussar S, Griffin NW, Bezy O, Fujisaka S, Vienberg S, Softic S, Deng L, Bry L, Gordon JI, Kahn CR. Interactions between Gut Microbiota, Host Genetics and Diet Modulate the Predisposition to Obesity and Metabolic Syndrome. Cell Metab 2015; 22:516-530

89. Vaarala O, Alfthan G, Jauhiainen M, Leirisalo-Repo M, Aho K, Palosuo T. Crossreaction between antibodies to oxidised low-density lipoprotein and to cardiolipin in systemic lupus erythematosus. Lancet 1993; 341:923-925

90. van der Meulen TA, Harmsen H, Bootsma H, Spijkervet F, Kroese F, Vissink A. The microbiome-systemic diseases connection. Oral Dis. 2016; 22:719-734

91. van loveren C. Sugar alcohols: what is the evidence for caries-preventive and caries-therapeutic effects? Caries Res. 2004; 38:286-293

92. van loveren C. Oral and dental health: prevention of dental caries, erosion, gingivitis and periodontitis. ILSI Europe Concise Monograph series 2009;

93. Wang C, Kankaanpaa J, Kummu O, Turunen SP, Akhi R, Bergmann U, Pussinen P, Remes AM, Horkko S. Characterization of a natural mouse monoclonal antibody recognizing epitopes shared by oxidized lowdensity lipoprotein and chaperonin 60 of Aggregatibacter actinomycetemcomitans. Immunol.Res. 2016; 64:699-710

94. Wang J, Qi J, Zhao H, He S, Zhang Y, Wei S, Zhao F. Metagenomic sequencing reveals microbiota and its functional potential associated with periodontal disease. Sci.Rep. 2013; 3:1843

95. Wegner N, Wait R, Sroka A, Eick S, Nguyen KA, Lundberg K, Kinloch A, Culshaw S, Potempa J, Venables PJ. Peptidylarginine deiminase from Porphyromonas gingivalis citrullinates human fibrinogen and alphaenolase: implications for autoimmunity in rheumatoid arthritis. Arthritis Rheum. 2010; 62:2662-2672

96. Wu J, Peters BA, Dominianni C, Zhang Y, Pei Z, Yang L, Ma Y, Purdue MP, Jacobs EJ, Gapstur SM, Li H, Alekseyenko AV, Hayes RB, Ahn J. Cigarette smoking and the oral microbiome in a large study of American adults. ISME.J. 2016; 
Oral Microbiome: Metagenomics in Oral Health

97. Yang F, Zeng X, Ning K, Liu KL, Lo CC, Wang W, Chen J, Wang D, Huang R, Chang X, Chain PS, Xie G, Ling J, Xu J. Saliva microbiomes distinguish caries-active from healthy human populations. ISME.J. 2012; 6:1-10

98. Zaura E, Brandt BW, Teixeira de Mattos MJ, Buijs MJ, Caspers MP, Rashid MU, Weintraub A, Nord CE, Savell A, Hu Y, Coates AR, Hubank M, Spratt DA, Wilson M, Keijser BJ, Crielaard W. Same Exposure but Two Radically Different Responses to Antibiotics: Resilience of the Salivary Microbiome versus Long-Term Microbial Shifts in Feces. MBio. 2015; 6:e01693-15

99. Zheng W, Zhang Z, Liu C, Qiao Y, Zhou D, Qu J, An H, Xiong M, Zhu Z, Zhao X. Metagenomic sequencing reveals altered metabolic pathways in the oral microbiota of sailors during a long sea voyage. Sci.Rep. 2015; 5:9131

Citation: Ling LU, Clémentine THABUIS, "Oral Microbiome: Metagenomics in Oral Health". American Research Journal of Dentistry; 1(1): 23-38.

Copyright (C) Ling LU, Clémentine THABUIS, This is an open access article distributed under the Creative Commons Attribution License, which permits unrestricted use, distribution, and reproduction in any medium, provided the original work is properly cited. 